013 PRIMARY CARE DIRECT ACCESS CORONARY ARTERY CALCIUM (CAC) SCORE - PROGNOSIS FROM A NOVEL SERVICE

J Tan, G Lewis, J Townend, B Holloway. University Hospitals Birmingham (UHB) NHS Foundation Trust, UK

\subsection{6/heartjnl-2016-309680.13}

Introduction "NICE guidelines 95: Chest pain of recent onset" states that in a clinically low risk patient, negative CAC study alone excludes obstructive coronary disease. We describe a novel service which allows primary care direct access referral for CT CAC, established at UHB in 2011. Our study aims to evaluate the prognostic value of a negative CT CAC within our centre.

Methods A retrospective search of the Radiology Information Solution (RIS) system was performed for all patients referred between October 2011 to December 2013. Each report was reviewed on RIS to determine negative studies. Only patients above 35 years who fit the criteria for low risk $(<29 \%)$ were included. Scans were performed on a dual source CT scanner without prior premedication. Outcome data on major adverse cardiac events (MACE) from the negative studies were obtained via the patient's electronic records and Office of National Statistics. The follow-up period was $2-4$ years.

Results 407 patients had CAC studies. 267 patients with a zero CAC score were included. There were no cases of coronaryrelated deaths, coronary revascularisation or hospitalisation secondary to acute coronary syndrome (ACS). 14/267 (4.9\%) patients re-attended the hospital via emergency department with chest pain but had ACS ruled out. 3/267 (1.1\%) deaths occurred with none attributed to cardiovascular disease.

Conclusion Our study suggests that a negative CAC score in a population referred directly from primary care for exclusion of significant coronary disease in line with NICE guidance 95 is associated with a low incidence of cardiovascular morbidity and mortality.

\section{PATTERNS OF EARLY ATHEROSCLEROSIS FORMATION AND CARDIAC REMODELLING IN HEALTHY ADULTS OF SOUTH ASIAN AND EUROPEAN DESCENT}

1Jonathan Weir-McCall, 'Deirdre B Cassidy, 'Jill JF Belch, ${ }^{2}$ Stephen J Gandy, ${ }^{1} \mathrm{~J}$ Graeme Houston, ${ }^{1}$ Matthew A Lambert, ${ }^{1}$ Roberta Littleford, ${ }^{1}$ Janice Rowland, ${ }^{1}$ Allan D Struthers, ${ }^{1}$ Faisel Khan. 'Division of Cardiovascular \& Diabetes Medicine, Ninewells Hospital, Dundee, UK; ${ }^{2}$ NHS Tayside Medical Physics, Ninewells Hospital, Dundee, UK

\subsection{6/heartjnl-2016-309680.14}

Introduction South Asians (SAs) have a higher risk of cardiovascular disease (CVD) and stroke, but paradoxically lower prevalence of peripheral arterial disease (PAD) than Western Europeans (WEs). The aim of this study was to determine early changes in systemic atherosclerotic burden and cardiac remodelling as measured using whole body cardiovascular MRI (WBCVMR).

Methods 19 SA and 38 age, gender and BMI matched WE were recruited. All were $\geq 40$ years, free from CVD and with a 10 year risk of CVD $<20 \%$. WB-CVMR was performed which comprised a whole body angiogram (WBA) and cardiac magnetic resonance (CMR). These were performed on a 3T MRI scanner following dual phase injection of gadoteric acid. A standardised atherosclerotic score (SAS) was calculated from the WBA, while indexed left ventricular mass and volumes were calculated from the CMR.
Results SAs exhibited a significantly lower iliofemoral atheroma burden (regional SAS $0.0 \pm 0.0$ vs $1.9 \pm 6.9, \mathrm{p}=0.048$ ) and a trend towards lower overall atheroma burden (WB SAS $0.7 \pm$ 0.8 vs $1.8 \pm 2.3, \mathrm{p}=0.1)$. They had significantly lower indexed left ventricular mass $\left(46.9 \pm 11.8\right.$ vs $56.9 \pm 13.4 \mathrm{ml} / \mathrm{m}^{2}, \mathrm{p}=$ $0.008)$, end diastolic volume $\left(63.9 \pm 10.4\right.$ vs $75.2 \pm 11.4 \mathrm{ml} / \mathrm{m}^{2}$, $\mathrm{p}=0.001)$, end systolic volume $(20.5 \pm 6.1 \mathrm{vs} 24.6 \pm 6.8 \mathrm{ml} /$ $\left.\mathrm{m}^{2}, \mathrm{p}=0.03\right)$ and stroke volume $(43.4 \pm 6.6 \mathrm{vs} 50.6 \pm 7.9 \mathrm{ml} /$ $\mathrm{m}^{2}, \mathrm{p}=0.001$ ), but with no significant difference in functional indices.

Conclusion South Asians have a lower peripheral atherosclerotic burden and smaller hearts than Western Europeans even in a healthy population. Thus the paradoxical high risk of CVD compared with PVD risk may be due to an adverse cardiac haemodynamic status incurred by the smaller heart rather than atherosclerosis.

\section{PREVALENCE, PATTERN AND SIGNIFICANCE OF LATE GADOLINIUM ENHANCEMENT IN A HEALTHY ASYMPTOMATIC COHORT}

${ }^{1}$ Jonathan R Weir-McCall, ${ }^{1}$ Kerrie Fitzgerald, ${ }^{1} \mathrm{C}$ Papagiorcopulo, ${ }^{2}$ Stephen J Gandy, ${ }^{1}$ Matthew Lambert, ${ }^{1} \mathrm{Jill}$ JJF Belch, ${ }^{2}$ Ian Cavin, ${ }^{1}$ Roberta Littleford, ${ }^{2}$ Jennifer A MacFarlane, ${ }^{1}$ Shona Z Matthew, ${ }^{2} \mathrm{R}$ Stephen Nicholas, ${ }^{1}$ Allan D Struthers, ${ }^{3}$ Frank Sullivan, ${ }^{2}$ Shelley A Waugh, ${ }^{4}$ Richard D White, ${ }^{1} \mathrm{~J}$ Graeme Houston. ${ }^{1}$ Department of Cardiovascular and Diabetes Medicine, College of Medicine, University of Dundee; ${ }^{2}$ NHS Tayside Medical Physics, Ninewells Hospital, Dundee; ${ }^{3}$ Department of Research and Innovation, North York General Hospital, University of Toronto; ${ }^{4}$ Department of Clinical Radiology, University Hospital of Wales, Cardiff

\subsection{6/heartjnl-2016-309680.15}

Introduction Unrecognised myocardial infarctions (UMIs) have been described in 19-30\% of the population using late gadolinium enhancement (LGE). However these studies have focussed on unselected cohorts including those with known cardiovascular disease. The aim of the current study was to ascertain the prevalence of UMIs in a non-high risk population and their physiological significance.

Methods 5,000 volunteers $>40$ years with no history of cardiovascular disease (CVD) and a 10 year risk of CVD of less than 20\% were recruited to the Tayside Screening for Cardiac Events (TASCFORCE) study. Those with a BNP level greater than their gender-specific median were invited for a whole-body MR angiogram and cardiac MR including LGE. LGE was classed as absent, UMI, or non-specific.

Results 1,529 completed the imaging study with 53 (3.6\%) excluded due to missing data or inadequate LGE image quality. 10 of the remaining $1476(0.67 \%)$ displayed LGE. Of these, 3 $(0.2 \%)$ were consistent with UMI, while 7 were non-specific occurring in the mid-myocardium $(n=4)$, epicardium $(n=1)$ or right ventricular insertion points $(\mathrm{n}=2)$. Those with UMI had significantly higher $\mathrm{BNP}($ median 116 (range 31-133) vs $22.6(5-175) \mathrm{pg} / \mathrm{ml}, \mathrm{p}=0.015)$, lower ejection fraction $(54.6$ $(36-62)$ vs $68.9(38-89) \%, p=0.007)$ and larger end systolic volume (36.3 (27-61) vs $\left.21.7(5-65) \mathrm{ml} / \mathrm{m}^{2}, \mathrm{p}=0.014\right)$. Those with non-specific LGE had lower diastolic blood pressure (68 (54-70) vs $72(46-98) \mathrm{mmHg}, \mathrm{p}=0.013)$, but no differences in their cardiac function.

Conclusion Despite previous reports describing high prevalence of UMI, those who are of low-intermediate cardiovascular risk have a very low prevalence of UMI. LGE typical of UMI is associated with significantly impaired cardiac function, while LGE atypical of UMI has no adverse effect on function. 